\title{
The Effects of Augmented Somatosensory Feedback on Postural Sway and Muscle Co-contraction in Different Sensory Conditions
}

\author{
Seo-hyun Kim¹, BPT, PT, Kyung-eun Lee ${ }^{1}$, BPT, PT, One-bin Lim², PhD, PT, Chung-hwi Yi², PhD, PT \\ ${ }^{1}$ Department of Physical Therapy, The Graduate School, Yonsei University, ${ }^{2}$ Department of Physical Therapy, College of Health Science, \\ Yonsei University, Wonju, Korea
}

\author{
Article Info \\ Received January 22, 2020 \\ Revised February 27, 2020 \\ Accepted March 13, 2020 \\ Corresponding Author \\ Chung-hwi Yi \\ E-mail: pteagle@yonsei.ac.kr \\ https://orcid.org/0000-0003-2554-8083
}

\section{Key Words \\ Feedback \\ Postural balance \\ Posture \\ Proprioception}

Background: Augmented somatosensory feedback stimulates the mechanoreceptor to deliver information on bodily position, improving the postural control. The various types of such feedback include ankle-foot orthoses (AFOs) and vibration. The optimal feedback to mitigate postural sway remains unclear, as does the effect of augmented somatosensory feedback on muscle co-contraction.

Objects: We compared postural sway and ankle muscle co-contraction without feedback (control) and with either of two forms of somatosensory feedback (AFOs and vibration).

Methods: We recruited 15 healthy subjects and tested them under three feedback conditions (control, AFOs, vibration) with two sensory conditions (eyes open, or eyes closed and the head tilted back), in random order. Postural sway was measured using a force platform; the mean sway area of the $95 \%$ confidence ellipse (AREA) and the mean velocity of the center-of-pressure displacement (VEL) were assessed. Co-contraction of the tibialis anterior and gastrocnemius muscles was measured using electromyography and converted into a cocontraction index $(\mathrm{Cl})$.

Results: We found significant main effects of the three feedback states on postural sway (AREA, VEL) and the Cl. The two sensory conditions exerted significant main effects on postural sway (AREA and VEL). AFOs reduced postural sway to a level significantly lower than that of the control $(p=0.014, p<0.001)$ or that afforded by vibration $(p=0.024, p<0.001)$. In terms of $\mathrm{Cl}$ amelioration, the AFOs condition was significantly better than the control ( $p$ $=0.004)$. Vibration did not significantly improve either postural sway or the $\mathrm{Cl}$ compared to the control condition. There was no significant interaction effect between the three feedback conditions and the two sensory conditions.

Conclusion: Lower-extremity devices such as AFOs enhance somatosensory perception, improving postural control and decreasing the $\mathrm{Cl}$ during static standing.

\section{INTRODUCTION}

Postural control is regulated by the visual, vestibular, and somatosensory systems ('somatosensory' refers to both cutaneous sensation and proprioception) [1]. During static standing, the most sensitive information on body sway is provided by proprioceptive musculoarticular cues (somatosensory cues from the lower extremities) [2-5]. Postural sway reflects poor coordination of musculoskeletal movements regulated by sensory and muscular feedback that are intended to maintain the standing balance [6-8]. Postural sway is most often characterized by displacements of the center of pressure (CoP), which reflects the orientation of body segments, and bodily movements that seek to preserve the center of gravity [9].

In the diminishing of sensory and muscular feedback, alternate sources of sensation substitute for the missing information. For example, a light fingertip touch on a stationary external surface reduces postural sway [10-12]. Somatosensory feedback yields positional information, contributing to selfperception of bodily motion via mechanical stimulation of cutaneous mechanoreceptors. Such feedback can be exploited to induce desired changes in muscle contraction patterns; muscles respond to ankle-foot orthoses (AFOs) and vibration [13-15]. 
Although the effects of AFOs on balance remain controversial, a recent systematic review suggested that postural control improved if the ankle range of motion was not limited [16]. Smalley et al. [15] found that AFOs reduced postural sway but footplates did not. In addition, during static standing, vibration applied bilaterally over the Achilles tendons imparted the illusion of lengthening of dorsiflexor muscles, which are the primary muscles that control postural sway [17]. The illusion enhances the sensitivity of leg muscle spindles, improving postural sway [13].

In order to examine only the somatosensory system in the postural balance, a certain sensory condition could be used to reduce the effects of visual and vestibular system by closing eyes and tilting head back [18]. And the effects of the somatosensory feedback can be better examined in the sensory condition (eye closed and head tilted back) that is greatly affected by the somatosensory system than in a normal sensory condition with visual, vestibular and somatosensory systems [15].

Although augmented somatosensory feedback improves postural sway by changing muscle contraction patterns, few studies have explored the activities of ankle postural muscles. Subjects lacking appropriate regulation of balance feedback use co-contraction of the postural muscles to excess while standing, as a compensation [19,20]. Co-contraction of ankle joint muscles increases joint stiffness and may restrict postural control [21,22]. In high ankle joint stiffness, body movement during static standing was less characteristic of an inverted pendulum and more reliant on hip/mixed strategies which induce the large postural sway [23]. This may increase the risk of falling; it is essential to ensure that augmented somatosensory feedback does not induce such effects.

AFOs and vibration improve postural sway; however, it remains unclear which form of feedback is optimal. No study has yet explored the effect of somatosensory feedback on ankle muscle co-contraction. Thus, the aim of this study was to compare the effects of feedback-free, AFOs and vibration on postural sway and muscle co-contraction with different sensory conditions. We hypothesized that augmented somatosensory feedback (AFOs and vibration) would reduce postural sway (the AREA and VEL parameters) and affect the co-contraction index (CI) during static standing.

\section{MATERIALS AND METHODS}

\section{Subjects}

Fifteen young age subjects (11 males, 4 females; mean age: $22.9 \pm 1.7$ years, height: $169.5 \pm 6.8 \mathrm{~cm}$, weight: $68.1 \pm 10.6$ $\mathrm{kg}$ ) participated in the study. All lacked any neuromuscular balance disorder, had no history of knee or ankle surgery, and did not feel dizzy when standing. Before participating in the study, all subjects were informed of the study procedure and methods. This study was approved by the Yonsei University Wonju Institutional Review Board (approval No. 1041849201910-BM-144-02).

\section{Instruments}

\section{1) Static postural sway testing}

A force platform (FDM S; Zebris Medical GmbH, Isny, Germany) was used to measure postural sway during static standing; signals were sampled at $100 \mathrm{~Hz}$. CoP displacements were employed to extract the mean sway area of the $95 \%$ confidence ellipse $\left(\mathrm{AREA}, \mathrm{cm}^{2}\right.$ ) and the mean velocity of CoP displacements (VEL, mm/s) [20]. The data were processed and verified using MR3 ver. 3.6.32 software (Noraxon Inc., Scottsdale, AZ, USA), which also synchronized and verified the electromyography (EMG) data.

\section{2) Electromyography recording}

The postural muscle activity of the ankle joint during static standing was measured using surface EMG (Noraxon TeleMyo DTS; Noraxon Inc.) at a sampling rate of 1,000 Hz. The tibialis anterior (TA) and gastrocnemius (GCM) muscle activities were recorded to explore ankle muscle co-contraction during static standing. Two unipolar electrodes were attached to each muscle, as recommended by Criswell [24]. For the TA muscle, two electrodes $2 \mathrm{~cm}$ apart were placed parallel to and just lateral to the medial shaft of the tibia (on the muscle belly); electrodes were placed directly on the GCM muscles [24]. Each TA and GCM EMG signal was normalized to the maximal signal obtained during static standing.

\section{3) Muscle co-contraction analysis}

EMG data were post-procced using Matlab (Matlab R2019a; MathWorks, Natick, MA, USA). Raw EMG signals were bandpass filtered at $15-500 \mathrm{~Hz}$ using a second-order Butterworth digital filter to remove noise or movement interference [20]. To 
explore the extent of TA and GCM co-contraction, the CI was calculated using the method of Falconer and Winter [25] employing the following equation: $C I=\frac{2 I_{\text {ant }}}{I_{\text {total }}} \times 100 \%$

$I_{\text {ant }}$ is the area of the total antagonistic activity calculated as $I_{\text {ant }}=\int_{t_{1}}^{t_{2}} E M G_{T A}(t) d t+\int_{t_{2}}^{t_{3}} E M G_{G C M}(t) d t$

where $t_{1}$ to $t_{2}$ is the interval during which the TA EMG signal is lower than the GCM signal and $t_{2}$ to $t_{3}$ is the opposite interval. $I_{\text {total }}$ is the integral of the summed TA and GCM EMG signals during task performance: $I_{\text {total }}=\int_{t_{1}}^{t_{3}}\left[E M G_{T A}+E M G_{G C M}\right](t) d t$

The higher the CI value, the greater the use of muscle cocontraction [25].

\section{4) Augmented somatosensory feedback}

AFOs (AOS-102; DOUM Medical, Seoul, Korea) were fabricated from $40 \mathrm{~mm}$ wide padded Velcro strapping consistent with the type used most frequently to secure orthoses. The flexible AFOs didn't restrict ankle range of motion in order not to directly affect the postural balance.

Vibration was delivered by two commercial vibrator (Magic Touch; Xiamen Lefan Wellness Science \& Technology Co., Ltd, Xiamen City, Fujian Province, China). Each vibrator was circular with a diameter of $5 \mathrm{~cm}$ with a flexible pad to fit over the Achilles tendon and was set from 0 to $100 \mathrm{~Hz}$. The vibration was applied continuously for one test.

\section{Procedure}

The subjects stand barefoot on the force platform with their feet together and their arms relaxed on both sides of the body. They were instructed to try not to sway during measurements taken over $60 \mathrm{~s}$ during all tests. Each subject was then asked to perform postural tasks with two sensory conditions: Eyes opened (EO), or eyes closed, and head tilted back (ECHB). Un- der the EO condition, the subjects stared at a stationary sign on paper placed $2 \mathrm{~m}$ from the front of the platform. Under the ECHB condition, the subjects stared at a sign on the ceiling about $30 \mathrm{~cm}$ in front of the platform and then closed their eyes. The ECHB condition reduced influence of visual system and vestibular system to isolate the somatosensory control of postural control [7]. Three feedback conditions were tested in each sensory condition: 1) control, no AFOs or vibration (Figure 1A), 2) AFOs, Wearing the AFOs on both lower legs (Figure 1B), 3) Vibration, vibrators on both Achilles tendon (Figure 1C). The tests were performed in random order using a randomization sequence created by Microsoft Excel (Microsoft Corp., Roselle, IL, USA). The subjects sat for 60 seconds (rest periods) between each test.

\section{Statistical Analysis}

The one-sample Kolmogorov-Smirnov test confirmed that the data were normally distributed. A $3 \times 2$ (three feedback conditions $\times$ two sensory conditions) repeated-measures analysis of variance was used to compare the CoP and EMG data; the Bonferroni correction was applied during post-hoc testing. A p-value $<0.05$ was taken to indicate statistical significance. SPSS software ver. 25 (IBM Corp., Armonk, NY, USA) was used for all statistical analyses.

\section{RESULTS}

The AREA, VEL, and CI values derived under the three feedback conditions in both positions are listed in Table 1.

\section{Postural Sway}

No significant (three feedback conditions $\times$ two sensory conditions) interaction was evident for either AREA ( $F=0.395$,
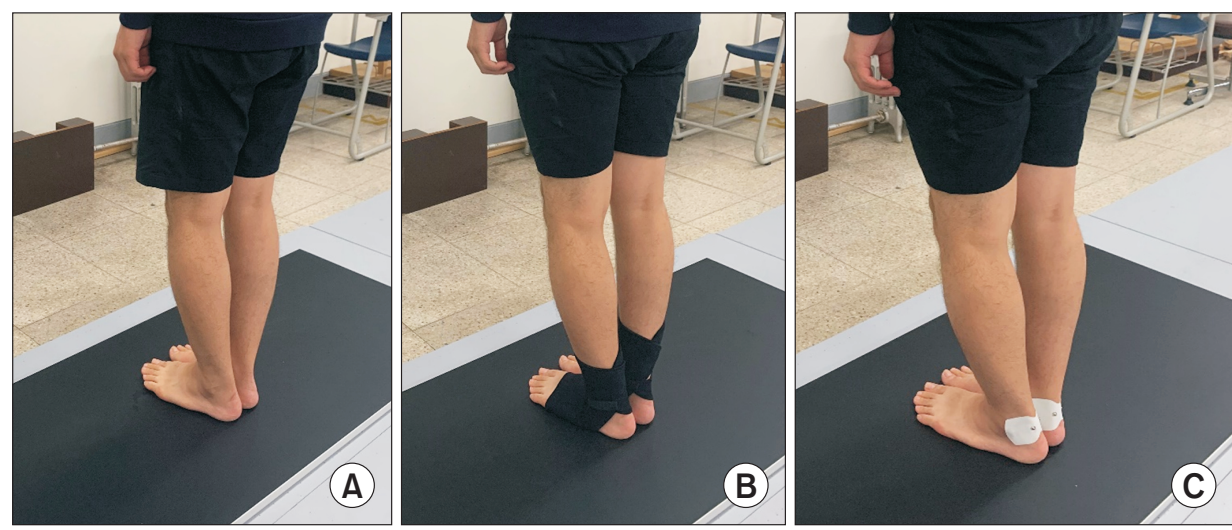

Figure 1. The three feedback conditions (A: control, B: AFOs, C: vibration). AFOs, ankle-foot orthoses. 
Table 1. Postural control and $\mathrm{Cl}$ data derived under the three feedback conditions $(\mathrm{N}=15)$

\begin{tabular}{|c|c|c|c|c|c|c|}
\hline \multirow{2}{*}{ Variables } & \multicolumn{2}{|c|}{ Control } & \multicolumn{2}{|c|}{ AFOs } & \multicolumn{2}{|c|}{ Vibration } \\
\hline & EO & $\mathrm{ECHB}$ & EO & $\mathrm{ECHB}$ & EO & $\mathrm{ECHB}$ \\
\hline AREA $\left(\mathrm{cm}^{2}\right)$ & $721.8 \pm 363.8$ & $1,340.1 \pm 777.1$ & $551.5 \pm 250.0$ & $838.5 \pm 314.7$ & $714.7 \pm 411.8$ & $1,152.1 \pm 540.5$ \\
\hline VEL $(\mathrm{mm} / \mathrm{s})$ & $12 \pm 3.0$ & $20.4 \pm 6.2$ & $9.9 \pm 2.6$ & $15.9 \pm 3.2$ & $11.7 \pm 3.5$ & $20.6 \pm 4.1$ \\
\hline $\mathrm{Cl}(\%)$ & $36.5 \pm 8.5$ & $39.3 \pm 9.2$ & $29.3 \pm 9.5$ & $35.4 \pm 9.7$ & $35.7 \pm 11.5$ & $36.4 \pm 8.4$ \\
\hline
\end{tabular}

Values are presented as mean \pm standard deviation. $\mathrm{Cl}$, co-contraction index, AFOs, ankle foot orthoses; EO, eye open; ECHB, eye closed and head tilted back; AREA, the mean sway area of the $95 \%$ confidence ellipse, VEL, the mean velocity of the center of pressure displacements.

Table 2. ANOVA analyses by two positions and three feedback conditions

\begin{tabular}{|c|c|c|c|c|c|c|}
\hline \multirow{2}{*}{ Variables } & \multicolumn{2}{|c|}{ Feedback } & \multicolumn{2}{|c|}{ Sensory } & \multicolumn{2}{|c|}{ Feedback $\times$ Sensory } \\
\hline & $\mathrm{F}$ & $\mathrm{p}$-value & $\mathrm{F}$ & $\mathrm{p}$-value & $\mathrm{F}$ & $\mathrm{p}$-value \\
\hline AREA & 0.680 & $0.005^{*}$ & 1.004 & $0.002 *$ & 0.395 & 0.131 \\
\hline VEL & 1.061 & $<0.001 *$ & 2.380 & $<0.001 *$ & 0.422 & 0.100 \\
\hline $\mathrm{Cl}$ & 0.747 & $0.002 *$ & 0.451 & 0.114 & 0.279 & 0.349 \\
\hline
\end{tabular}

AREA, the mean sway area of the $95 \%$ confidence ellipse; VEL, the mean velocity of the center of pressure displacements; $\mathrm{Cl}$, co-contraction index. ${ }^{*} \mathrm{p}<$ 0.05 .
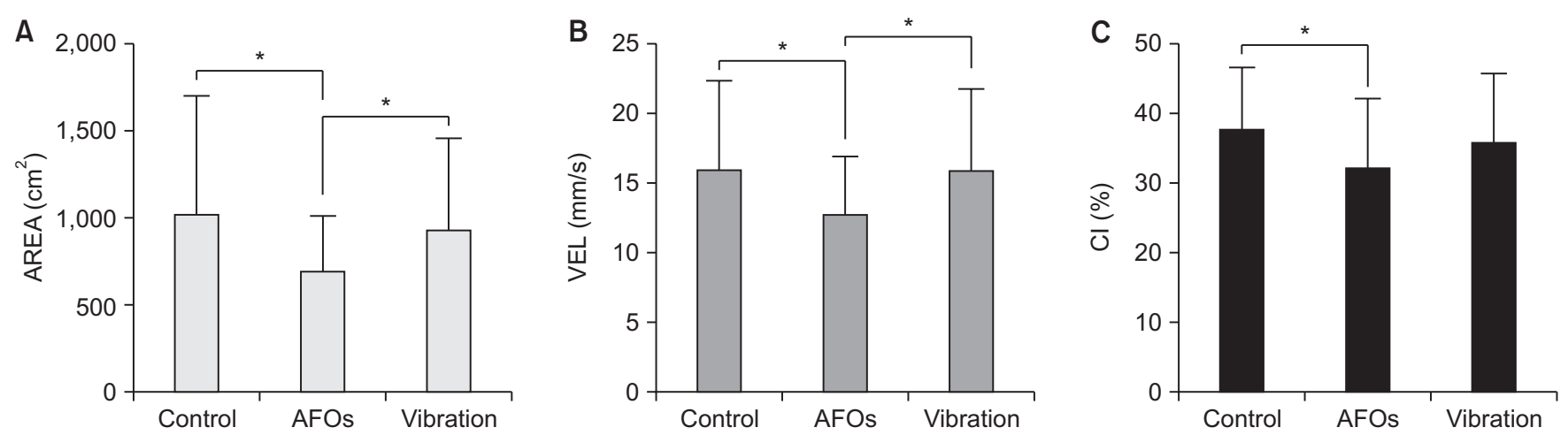

Figure 2. Post-hoc comparisons of the effects of the three feedback conditions (control, AFOs, and vibration) on the (A) AREA, (B) VEL, and (C) Cl values during static standing. AREA, the mean sway area of the $95 \%$ confidence ellipse; VEL, the mean velocity of the center of pressure displacements; $\mathrm{Cl}$, cocontraction index; AFOs, ankle-foot orthoses. ${ }^{*} p<0.05$.

$\mathrm{p}=0.131)$ or VEL $(\mathrm{F}=0.422, \mathrm{p}=0.100)$. In three feedback conditions (control, AFOs, and vibration), significant main effects on AREA ( $F=1.004, p=0.002)$ and VEL $(F=2.380, p<0.001)$ were apparent (Table 2). AFOs had significant difference with the control $(p=0.014)$ and vibration $(p=0.024)$ in AREA (Figure $2 \mathrm{~A}$ ). In addition, AFOs had significant difference with the control ( $p<0.001)$ and vibration $(p<0.001)$ in VEL (Figure 2B). Vibration (compared to the control condition) did not exhibit any significant effects on AREA or VEL. Significant main effects of the two sensory conditions (EO and ECHB) on AREA ( $F=$ 0.680, $p=0.005)$ and VEL $(F=1.061, p<0.001)$ were apparent (Table 2). AREA and VEL of postural sway were had significant increase under the ECHB condition than under the EO condition $(\mathrm{p}=0.002, \mathrm{p}<0.001)($ Figure $3 \mathrm{~A}, \mathrm{~B})$.

\section{Ankle Muscle Co-contraction}

There was no significant interaction effect in terms of CI (F $=0.279, \mathrm{p}=0.349)$. The three type of feedback exerted significant main effects on $\mathrm{CI}(\mathrm{F}=0.747, \mathrm{p}=0.002)$ (Table 2). The AFOs significantly decreased the CI compared to the control condition ( $p=0.004)$, but the effects of AFOs and vibration did not differ significantly $(p=0.133)$. The effect of vibration did not differ significantly from that of the control condition $(\mathrm{p}=$ 0.403 ) (Figure $2 \mathrm{C}$ ). When comparing the two sensory conditions, no significant main effect on $\mathrm{CI}$ was evident $(\mathrm{F}=0.451, \mathrm{p}$ $=0.0114$ ) (Figure 3C).

\section{DISCUSSION}

AFOs and vibration were used to improve the postural 

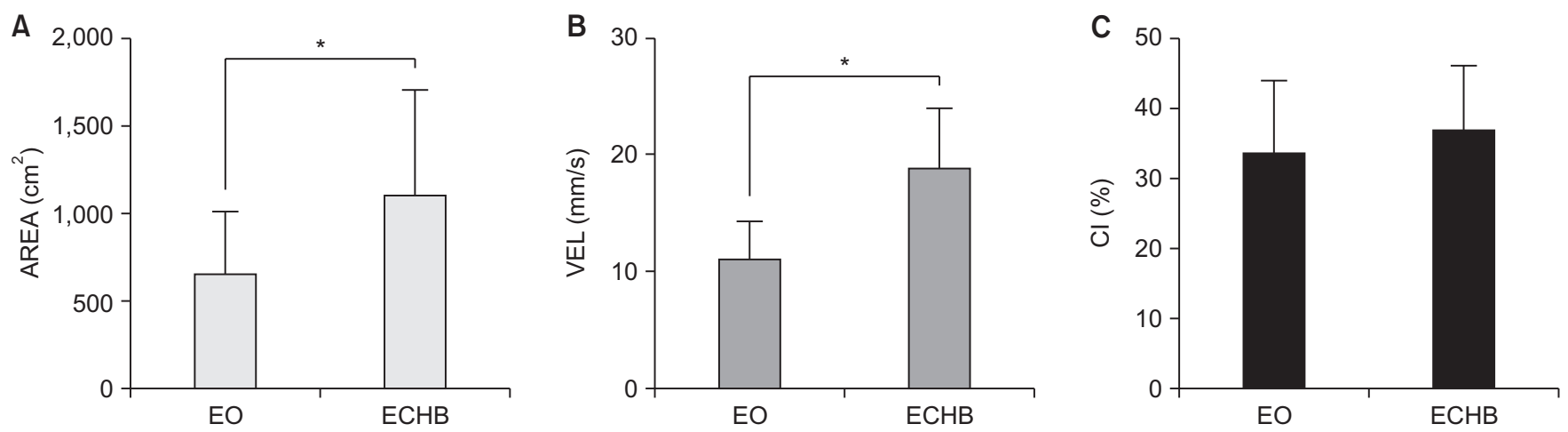

Figure 3. Post-hoc comparisons of the two positions leyes open [EO], eyes closed with the head tilted back [ECHB]) on the (A) AREA, (B) VEL, and (C) Cl values during static standing. AREA, the mean sway area of the $95 \%$ confidence ellipse; VEL, the mean velocity of the center of pressure displacements; $\mathrm{Cl}$, co-contraction index. ${ }^{*} \mathrm{p}<0.05$.

control. However, few studies have explored postural muscle co-contraction during augmented somatosensory feedback. Therefore, the aim of this study was to investigate whether postural sway (AREA and VEL) and CI changed during three feedback conditions delivered to subjects in two sensory conditions. The result suggested that AFOs not only improve postural control but also reduce postural muscle co-contraction. The AREA, VEL data revealed significant main effect of three feedback conditions; AFOs were better than both the control and vibration conditions. As the AFOs contact greater leg area than the vibrations, AFOs may deliver more sensory information. Menz et al. [26] founded that more proximal stimuli were more effective in reducing postural sway. The present study supports these results since the somatosensory feedback from the AFOs could deliver more proximal cutaneous information than vibration. In fact, vibration did not significantly improve postural sway compared to the control, even though many previous studies found that vibration applied both Achilles tendon improve postural sway $[13,17,27,28]$. With the subjects standing, vibration at $80-100 \mathrm{~Hz}$ applied to the Achilles tendon activated I a spindle afferents, inducing postural control improvement [29,30]; the vibration in this study was not always within the range of 80-100 Hz. The effect of vibration was insufficient to make significant improvement of postural sway.

During static standing, the plantarflexor are continuously activated to maintain stable balance; the center of mass lies in front of the ankle joint. Dorsiflexors are rarely activated during static standing, but subjects with impaired postural control frequently engage the dorsiflexors. Both muscles must be appropriately activated to stabilize postural sway [31]. AFOs provide adequate somatosensory cues to both the dorsiflexors and the plantarflexors; the AFOs cover the entire lower legs. Thus, the postural muscles are alternately activated because both muscles receive enough bodily position information. On the other hand, vibration applied both Achilles tendon were received by only the plantarflexors. There was not enough information to control the dorsiflexors efficiently. Furthermore, somatosensory feedback stimulates mechanoreceptors, strengthening somatosensory perception and facilitating feedback that corrects directional changes. Enhanced sensitivity of bodily position awareness allows smaller counter-movements and result in less muscle activation $[7,8,32]$ The AFOs affording the greatest reductions in AREA and VEL values induced less body movement than other forms of feedback, reducing the need for ankle muscle activity, and, in turn, the CI value. Vibration did not significantly reduce the CI because it did not significantly improve either the AREA or VEL value.

We confirmed that two sensory conditions exerted main effect on AREA and VEL. A previous study founded no significant effect of somatosensory feedback in EO condition, but a significant effect in ECHB condition (Smalley et al. [15]). The EO condition is more associated with other sensory systems than ECHB condition; visual and vestibular cues deliver sensory information relevant to postural control [18]. However, we found no significant main effects of either sensory condition on the CI. The sensory conditions (EO, ECHB) differed in terms of the factors affecting postural sway; fewer useful cues were available in the ECHB condition than the EO condition. Also, we found no interaction effects among the three feedback conditions and the two sensory conditions, when static standing, the somatosensory system comes to the forefront; the results thus tended to be similar under either sensory condition. 
Our study had several limitations. First, the vibrational frequency was not confined to $80-100 \mathrm{~Hz}$, which is thought to be optimal in terms of postural control. Second, in this study, we studied only the TA and GCM muscles; the soleus can also affect the postural control. However, because the vibrators were attached to the Achilles tendon, measures of soleus muscle activity may have created noise. Third, we did not explore dynamic balance, which is critical in daily life. Further research should explore postural muscle co-contraction with dynamic balance.

\section{CONCLUSIONS}

Our results suggest that AFOs of the somatosensory feedbacks improve postural sway and reduce ankle muscle co-contraction Such feedback stimulates muscle mechanoreceptors to enhance somatosensory awareness, rather than inducing excessive muscle contractions, and it is particularly sensitive to changes in body movements and position. Therefore, static balance training using flexible AFOs would effectively improve postural control while preventing excessive ankle muscle cocontraction during static standing. If further work shows that the effects persist even after removing the feedback, balance training would become more efficient.

\section{CONFLICTS OF INTEREST}

No potential conflict of interest relevant to this article was reported.

\section{AUTHOR CONTRIBUTIONS}

Conceptualization: CY. Data curation: SK, KL. Formal analysis: SK. Investigation: SK. Methodology: SK, OL. Project administration: SK, OL. Resources: SK, KL. Software: SK. Supervision: CY. Validation: SK. Visualization: SK. Writing - original draft: SK. Writing - review \& editing: SK.

\section{ORCID}

Seo-hyun Kim: https://orcid.org/0000-0002-6978-200X

Kyung-eun Lee: https://orcid.org/0000-0003-4021-9181

One-bin Lim: https://orcid.org/0000-0002-5281-3463

\section{REFERENCES}

1. Johansson R, Magnusson M. Human postural dynamics. Crit Rev Biomed Eng 1991;18(6):413-37.

2. Fitzpatrick R, Burke D, Gandevia SC. Task-dependent reflex responses and movement illusions evoked by galvanic vestibular stimulation in standing humans. J Physiol 1994;478(Pt 2):363-72.

3. Fitzpatrick R, McCloskey DI. Proprioceptive, visual and vestibular thresholds for the perception of sway during standing in humans. J Physiol 1994;478(Pt 1):173-86.

4. Goble DJ, Coxon JP, Wenderoth N, Van Impe A, Swinnen SP. Proprioceptive sensibility in the elderly: degeneration, functional consequences and plastic-adaptive processes. Neurosci Biobehav Rev 2009;33(3):271-8.

5. Goble DJ. Proprioceptive acuity assessment via joint position matching: from basic science to general practice. Phys Ther 2010;90(8):1176-84.

6. Blackburn T, Guskiewicz KM, Petschauer MA, Prentice WE. Balance and joint stability: the relative contributions of proprioception and muscular strength. J Sport Rehabil 2000;9(4):315-28.

7. Sarabon N, Rosker J, Loefler S, Kern H. The effect of vision elimination during quiet stance tasks with different feet positions. Gait Posture 2013;38(4):708-11.

8. Simoneau GG, Degner RM, Kramper CA, Kittleson KH. Changes in ankle joint proprioception resulting from strips of athletic tape applied over the skin. J Athl Train 1997;32(2):141-7.

9. Prieto TE, Myklebust JB, Hoffmann RG, Lovett EG, Myklebust BM. Measures of postural steadiness: differences between healthy young and elderly adults. IEEE Trans Biomed Eng 1996;43(9):956-66.

10. Baldan AM, Alouche SR, Araujo IM, Freitas SM. Effect of light touch on postural sway in individuals with balance problems: a systematic review. Gait Posture 2014;40(1):1-10.

11. Holden M, Ventura J, Lackner JR. Stabilization of posture by precision contact of the index finger. J Vestib Res 1994;4(4):285-301.

12. Jeka JJ, Lackner JR. Fingertip contact influences human postural control. Exp Brain Res 1994;100(3):495-502.

13. Borel L, Ribot-Ciscar E. Improving postural control by applying mechanical noise to ankle muscle tendons. Exp Brain Res 2016;234(8):2305-14.

14. Gross MT, Mercer VS, Lin FC. Effects of foot orthoses 
on balance in older adults. J Orthop Sports Phys Ther 2012;42(7):649-57.

15. Smalley A, White SC, Burkard R. The effect of augmented somatosensory feedback on standing postural sway. Gait Posture 2018;60:76-80.

16. Ramstrand N, Ramstrand S. AAOP state-of-the-science evidence report: the effect of ankle-foot orthoses on balance-a systematic review. J Prosthet Orthot 2010;22(10):P4-23.

17. Doumas M, Valkanidis TC, Hatzitaki V. Putting proprioception for balance to the test: contrasting and combining sway referencing and tendon vibration. Gait Posture 2019;67:201-6.

18. Simoneau GG, Ulbrecht JS, Derr JA, Cavanagh PR. Role of somatosensory input in the control of human posture. Gait Posture 1995;3(3):115-22.

19. Donath L, Kurz E, Roth R, Zahner L, Faude O. Different ankle muscle coordination patterns and co-activation during quiet stance between young adults and seniors do not change after a bout of high intensity training. BMC Geriatr 2015;15:19.

20. Maktouf W, Durand S, Boyas S, Pouliquen C, Beaune B. Combined effects of aging and obesity on postural control, muscle activity and maximal voluntary force of muscles mobilizing ankle joint. J Biomech 2018;79:198-206.

21. Butler AA, Lord SR, Rogers MW, Fitzpatrick RC. Muscle weakness impairs the proprioceptive control of human standing. Brain Res 2008;1242:244-51.

22. Ge W. Age-related differences in body segmental movement during perturbed stance in humans. Clin Biomech (Bristol, Avon) 1998;13(4-5):300-7.

23. Warnica MJ, Weaver TB, Prentice SD, Laing AC. The influence of ankle muscle activation on postural sway during quiet stance. Gait Posture 2014;39(4):1115-21.

24. Criswell E. Cram's introduction to surface electromyography. Sudbury: Jones \& Bartlett; 2010.

25. Falconer K, Winter DA. Quantitative assessment of co-contraction at the ankle joint in walking. Electromyogr Clin Neurophysiol 1985;25(2-3):135-49.

26. Menz HB, Lord SR, Fitzpatrick RC. A tactile stimulus applied to the leg improves postural stability in young, old and neuropathic subjects. Neurosci Lett 2006;406(1-2):23-6.

27. Aboutorabi A, Arazpour M, Bahramizadeh M, Farahmand F, Fadayevatan R. Effect of vibration on postural control and gait of elderly subjects: a systematic review. Aging Clin Exp Res 2018;30(7):713-26.

28. Ribot-Ciscar E, Hospod V, Aimonetti JM. Noise-enhanced kinaesthesia: a psychophysical and microneurographic study. Exp Brain Res 2013;228(4):503-11.

29. Burke D, Hagbarth KE, Löfstedt L, Wallin BG. The responses of human muscle spindle endings to vibration of non-contracting muscles. J Physiol 1976;261(3):673-93.

30. Roll JP, Vedel JP, Ribot E. Alteration of proprioceptive messages induced by tendon vibration in man: a microneurographic study. Exp Brain Res 1989;76(1):213-22.

31. Vette AH, Sayenko DG, Jones M, Abe MO, Nakazawa K, Masani K. Ankle muscle co-contractions during quiet standing are associated with decreased postural steadiness in the elderly. Gait Posture 2017;55:31-6.

32. Hijmans JM, Geertzen JH, Dijkstra PU, Postema K. A systematic review of the effects of shoes and other ankle or foot appliances on balance in older people and people with peripheral nervous system disorders. Gait Posture 2007;25(2):316-23. 